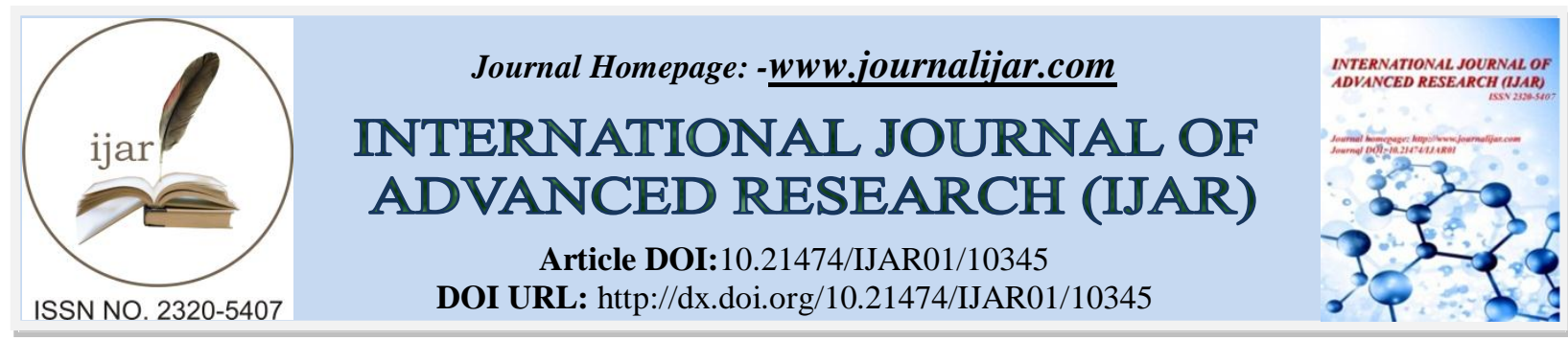

RESEARCH ARTICLE

\title{
VARIABILITY AND HERITABILITY STUDIES FOR YIELD AND YIELD COMPONENT TRAITS IN FOXTAIL MILLET
}

Dhruv Shukla, R. P. Joshi and Kartikey Sootrakar

Deptt. of Plant Breeding \& Genetics, JNKVV, College of Agriculture, Rewa, 486001.

\section{Manuscript Info}

Manuscript History

Received: 27 November 2019

Final Accepted: 30 December 2019

Published: January 2020

Key words:-

Foxtail Millet, Variability, Heritability, PCV, GCV

\begin{abstract}
The present investigation was carried out at All India Coordinated Research project on Small Millet, at Instructional Farm, College of Agriculture, Rewa (MP) during kharif 2017. Estimates of phenotypic coefficient of variation was found higher than their corresponding genotypic coefficient of variation, but for all the characters differences was found non - significant except tillers per plant indicating that the little influence of environment on the expression these characters. The moderate magnitude of phenotypic coefficient of variation (PCV) along with genotypic coefficient of variation $(\mathrm{GCV})$ was recorded for grain yield per plant, number of tillers per plant, harvest index and ear length. High heritability coupled with high genetic advance as percent of mean was observed for number of tillers per plant.
\end{abstract}

Copy Right, IJAR, 2020,. All rights reserved.

\section{Introduction:-}

Foxtail millet (Setaria italica L.) is one of the oldest crops cultivated for food grain, hay and pasture. It has the longest history of cultivation among the millets, having been grown in China since sometime in the sixth millennium BC. The geographical origin of foxtail millet based on cytological studies indicated that wild ancestor of foxtail millet is S. viridis ( $\mathrm{Li}$ et al. 1945). Knowledge on heritability and genetic advance of the character indicate the scope for the improvement of a trait through selection. Heritability estimates along with genetic advance are also helpful in predicting the gain under selection. Grain yield being a complex character is very difficult to improve by selecting the genotypes for yield per se, therefore identifying the characters which are closely related and have contributed to yield becomes highly essential.

\section{Material And Methods:-}

Present investigation was carried out at All India Coordinated Research project on Small Millet, at Instructional Farm, College of Agriculture Rewa (MP) during kharif 2017. The experimental materials of the present study comprised 16 ( DHFT 5-6, SiA3179, DHFT77-3, PPSS-7, PKS 22, SiA 3220, TNSi 337, SiA 3212, SiA 3219 , TNSi345, DHFT35-3, DHFT2-5-3, IIMR FtM-1, SiA 326, SiA 3156 and RFM 41) genotypes of Foxtail Millet. The parents were selected on the basis of different genetic and geographical origin.

Table 1:- Analysis of variance for 12 quantitative characters in Foxtail Millet.

\begin{tabular}{|c|c|c|c|c|c|c|c|c|c|c|c|c|c|}
\hline Sov & df & $\begin{array}{l}\text { Days } \\
\text { to } \\
50 \% \\
\text { flow }\end{array}$ & $\begin{array}{l}\text { Days } \\
\text { to } \\
\text { matur } \\
\text { ity }\end{array}$ & $\begin{array}{l}\text { Plan } \\
\text { t } \\
\text { heig } \\
\text { ht }\end{array}$ & $\begin{array}{l}\text { No. } \\
\text { of } \\
\text { Tille } \\
\text { rs/ }\end{array}$ & $\begin{array}{l}\text { Flag } \\
\text { leaf } \\
\text { leng } \\
\text { th }\end{array}$ & $\begin{array}{l}\text { Flag } \\
\text { leaf } \\
\text { widt } \\
\text { h }\end{array}$ & $\begin{array}{l}\text { Ped } \\
\text { uncl } \\
\text { e } \\
\text { lengt }\end{array}$ & $\begin{array}{l}\text { Ear } \\
\text { lengt } \\
\text { h } \\
\text { (cm) }\end{array}$ & $\begin{array}{l}\text { Biol } \\
\text { ogic } \\
\text { al } \\
\text { yield }\end{array}$ & $\begin{array}{l}\text { Harve } \\
\text { st } \\
\text { Index }\end{array}$ & $\begin{array}{l}1000 \\
\text { grain } \\
\text { weigh } \\
t\end{array}$ & $\begin{array}{l}\text { Gr } \\
\text { ain } \\
\text { yiel } \\
\text { d/ }\end{array}$ \\
\hline
\end{tabular}




\begin{tabular}{|c|c|c|c|c|c|c|c|c|c|c|c|c|c|}
\hline & & $\begin{array}{l}\text { erin } \\
\mathrm{g}\end{array}$ & & $(\mathbf{c m})$ & $\begin{array}{l}\text { plan } \\
\text { t }\end{array}$ & (cm) & $(\mathbf{c m})$ & $\begin{array}{l}\text { h } \\
(\mathbf{c m})\end{array}$ & & $\begin{array}{l}\text { I } \\
\text { plan } \\
\text { t (g) }\end{array}$ & & (g) & $\begin{array}{l}\text { pla } \\
\text { nt } \\
\text { (g) }\end{array}$ \\
\hline $\begin{array}{l}\text { Replica } \\
\text { tion }\end{array}$ & 2.00 & $\begin{array}{l}10.8 \\
9\end{array}$ & 4.00 & 63.48 & $\begin{array}{l}0.00 \\
25 \\
\end{array}$ & 1.34 & 0.01 & 5.73 & 1.00 & 1.10 & 33.84 & 0.04 & $\begin{array}{l}0.2 \\
0 \\
\end{array}$ \\
\hline $\begin{array}{l}\text { Treatm } \\
\text { ent }\end{array}$ & 15.00 & $\begin{array}{l}36.5 \\
7 * *\end{array}$ & $\begin{array}{l}121.0 \\
7 * *\end{array}$ & $\begin{array}{l}448.7 \\
* *\end{array}$ & $\begin{array}{l}0.10 \\
* *\end{array}$ & $\begin{array}{l}21.7 \\
* *\end{array}$ & $\begin{array}{l}0.08 \\
* *\end{array}$ & $\begin{array}{l}9.87 \\
* *\end{array}$ & $\begin{array}{l}20.94 \\
* *\end{array}$ & $\begin{array}{l}5.1^{*} \\
*\end{array}$ & $\begin{array}{l}81.72 * \\
*\end{array}$ & $\begin{array}{l}0.13 * \\
*\end{array}$ & $\begin{array}{l}2.2 \\
* *\end{array}$ \\
\hline Error & 30.00 & 6.38 & 19.88 & 151.7 & 0.01 & 7.12 & 0.02 & 3.68 & 3.64 & 2.45 & 24.79 & 0.02 & 0.5 \\
\hline
\end{tabular}

\section{Result And Discussion:-}

A wide range of phenotypic coefficient of variation (PCV) was observed for all the traits ranged from 7.91 to 17.77. Moderate magnitude of PCV was recorded for grain yield per plant (17.77) followed by number of tillers per plant (17.38), harvest index ( 16.66 ), ear length ( 14.56 ), biological yield ( 12.34 ), Plant height (10.70), flag leaf width (10.61), and flag leaf length (10.44), while low estimate of PCV was recorded by 1000 grain weight ( 9.42 ), days to maturity (9.03), days to $50 \%$ flowering (8.26), and peduncle length (7.91).

Genotypic coefficient of variation (GCV) ranged from 4.74 to 13.64 . Moderate magnitudes of GCV were recorded for number of tillers per plant (13.64) followed by grain yield per plant (12.56), ear length (11.40), harvest index (10.97) and lower estimate of GCV were recorded for 1000 grain weight (7.50), days to maturity (7.16), flag leaf width (6.73), plant height (6.72), flag leaf length (6.65), days to $50 \%$ flowering (6.46), biological yield per plant(6.36) and for peduncle length (4.74).

High estimates of heritability in broad sense were recorded for 1000 grain weight $(63 \%)$ followed by days to maturity (62\%), days to 50\% flowering (61\%), number of tillers per plant (61\%) and for ear length (61\%). The high values indicate that heritability may be due to higher contribution of genotypic component. Similarly moderate ranged heritability were recorded for grain yield per plant (49\%), harvest index (43\%), flag leaf length (40\%), flag leaf width (40\%), plant height (39\%),peduncle length (35\%) and lowest value was recorded for biological yield per plant $(26 \%)$.

A perusal of genetic advance revealed that it was high for number of tillers per plant (22.05) and moderate for ear length (18.38), grain yield per plant (18.30), harvest index (14.88), 1000 grain weight ( 12.32 ), days to maturity (11.70), and for days to $50 \%$ flowering (10.42). The lowest value of genetic advance as \% of mean observed for flag leaf width (8.80), flag leaf length (8.72), plant height (8.70), biological yield (6.75) and for peduncle length (5.84).

Moderate heritability was showed by grain yield per plant followed by harvest index, flag leaf length, flag leaf width, plant height and peduncle length. Previously moderate heritability for grain yield has also been reported by Patil et al.(1989) and for plant height by velzaco et al. (2012).

Table 2:- Estimation of genetic parameters for different quantitative characters in Foxtail Millet.

\begin{tabular}{|c|c|c|c|c|c|c|c|c|}
\hline \multirow[t]{2}{*}{ S.N. } & \multirow[t]{2}{*}{ Character } & \multirow[t]{2}{*}{ Mean } & \multicolumn{2}{|l|}{ Range } & \multirow[t]{2}{*}{ PCV } & \multirow[t]{2}{*}{ GCV } & \multirow{2}{*}{$\begin{array}{ll}\mathbf{h}^{2} & (\mathrm{bs}) \\
\% & \end{array}$} & \multirow{2}{*}{$\begin{array}{l}\text { GA as\% of } \\
\text { mean }\end{array}$} \\
\hline & & & Minimum & Maximum & & & & \\
\hline 1 & Days to $50 \%$ flowering & 49.04 & 42.00 & 54.33 & 8.26 & 6.46 & 61 & 10.42 \\
\hline 2 & Days to maturity & 82.62 & 70.66 & 90.66 & 9.03 & 7.16 & 62 & 11.70 \\
\hline 3 & Plant height & 147.8 & 128.6 & 167.0 & 10.70 & 6.72 & 39 & 8.70 \\
\hline 4 & No of tillers & 1.26 & 1.0 & 1.6 & 17.38 & 13.64 & 61 & 22.05 \\
\hline 5 & Flag leaf length & 33.13 & 26.03 & 36.20 & 10.44 & 6.65 & 40 & 8.72 \\
\hline 6 & Flag leaf width & 2.09 & 1.63 & 2.33 & 10.61 & 6.73 & 40 & 8.80 \\
\hline 7 & Peduncle length & 30.27 & 26.86 & 34.06 & 7.91 & 4.74 & 35 & 5.84 \\
\hline 8 & Ear length & 21.06 & 13.26 & 24.06 & 14.56 & 11.40 & 61 & 18.38 \\
\hline 9 & Biological Yield & 14.81 & 12.80 & 17.46 & 12.34 & 6.36 & 26 & 6.75 \\
\hline 10 & Harvest index & 39.70 & 29.66 & 47.23 & 16.66 & 10.97 & 43 & 14.88 \\
\hline 11 & 1000 grain weight & 2.58 & 2.00 & 2.83 & 9.42 & 7.50 & 63 & 12.32 \\
\hline
\end{tabular}




\begin{tabular}{|l|l|l|l|l|l|l|l|l|}
\hline 12 & Grain yield per plant & 5.93 & 4.76 & 8.00 & 17.77 & 12.56 & 49 & 18.30 \\
\hline
\end{tabular}

The estimate of genetic advance as percent of mean moderate for ear length followed by grain yield per plant, harvest index, days to maturity and days to $50 \%$ flowering. The moderate estimates of genetic advance as percentage of mean for days to $50 \%$ flowering were also reported by Patil et al.(1989).

High heritability coupled with high genetic advance as percent of mean was observed for number of tillers per plant. High values indicate that heritability may be due to higher contribution of genotypic component. The heritability estimates coupled with expected genetic advance indicate the additive mode of gene action in the expression of traits which helps in choosing an appropriate breeding methodology. Similar results were reported by Gurunadharao et al.(1984), Islam et al. (1990), prasanana et al.(2013) and Nirmalakumari et al.(2008).

Table 3:- Phenotypic and genotypic correlation between grain yield and its components in Foxtail Millet.

\begin{tabular}{|c|c|c|c|c|c|c|c|c|c|c|c|c|c|c|}
\hline $\begin{array}{l}\text { S. } \\
\text { N. }\end{array}$ & $\begin{array}{l}\text { Char } \\
\text { acters }\end{array}$ & & $\begin{array}{l}\text { Days } \\
\text { to } \\
50 \% \\
\text { flowe } \\
\text { ring }\end{array}$ & $\begin{array}{l}\text { Days } \\
\text { to } \\
\text { mat } \\
\text { urity }\end{array}$ & $\begin{array}{l}\text { Pla } \\
\text { nt } \\
\text { hei } \\
\text { ght }\end{array}$ & $\begin{array}{l}\text { Num } \\
\text { ber } \\
\text { of } \\
\text { tiller } \\
\text { s per } \\
\text { plant }\end{array}$ & $\begin{array}{l}\text { Flag } \\
\text { leaf } \\
\text { lengt } \\
h\end{array}$ & $\begin{array}{l}\text { Flag } \\
\text { leaf } \\
\text { widt } \\
\text { h }\end{array}$ & $\begin{array}{l}\text { Pedu } \\
\text { ncle } \\
\text { lengt } \\
\text { h }\end{array}$ & $\begin{array}{l}\text { Ear } \\
\text { lengt } \\
\text { h }\end{array}$ & $\begin{array}{l}\text { Biolo } \\
\text { gical } \\
\text { yield } \\
\text { per } \\
\text { plant }\end{array}$ & $\begin{array}{l}\text { Harv } \\
\text { est } \\
\text { Inde } \\
x\end{array}$ & $\begin{array}{l}\text { 1000 } \\
\text { grain } \\
\text { weig } \\
\text { ht }\end{array}$ & $\begin{array}{l}\text { Grai } \\
\text { n } \\
\text { yield } \\
\text { per } \\
\text { plant }\end{array}$ \\
\hline \multirow[t]{2}{*}{1} & \multirow{2}{*}{$\begin{array}{l}\text { Days } \\
\text { to } \\
50 \% \\
\text { flower } \\
\text { ing }\end{array}$} & $\mathrm{P}$ & 1 & $\begin{array}{l}0.62 \\
02 \\
* * *\end{array}$ & $\begin{array}{l}0.1 \\
767\end{array}$ & $\begin{array}{l}0.617 \\
9 * * *\end{array}$ & $\begin{array}{l}0.661 \\
2 * * *\end{array}$ & $\begin{array}{l}0.655 \\
7 * * *\end{array}$ & $\begin{array}{l}0.25 \\
70\end{array}$ & $\begin{array}{l}0.826 \\
3 * * *\end{array}$ & $\begin{array}{l}0.570 \\
6 * * *\end{array}$ & $\begin{array}{l}0.661 \\
5 * * *\end{array}$ & $\begin{array}{l}- \\
0.727 \\
7 * * *\end{array}$ & $\begin{array}{l}0.593 \\
0 * * *\end{array}$ \\
\hline & & $\mathrm{G}$ & 1 & $\begin{array}{l}0.73 \\
27\end{array}$ & $\begin{array}{l}0.4 \\
950\end{array}$ & $\begin{array}{l}0.834 \\
0\end{array}$ & $\begin{array}{l}0.784 \\
0\end{array}$ & $\begin{array}{l}0.806 \\
1\end{array}$ & $\begin{array}{l}0.40 \\
66\end{array}$ & $\begin{array}{l}0.931 \\
7\end{array}$ & $\begin{array}{l}0.711 \\
2\end{array}$ & $\begin{array}{l}0.767 \\
7\end{array}$ & $\begin{array}{l}- \\
0.915 \\
5\end{array}$ & $\begin{array}{l}0.168 \\
6\end{array}$ \\
\hline \multirow[t]{2}{*}{2} & \multirow{2}{*}{$\begin{array}{l}\text { Days } \\
\text { to } \\
\text { maturi } \\
\text { ty }\end{array}$} & $\mathrm{P}$ & & 1 & $\begin{array}{l}0.0 \\
814\end{array}$ & $\begin{array}{l}0.440 \\
1 * *\end{array}$ & $\begin{array}{l}0.337 \\
9^{*}\end{array}$ & $\begin{array}{l}0.518 \\
7 * * *\end{array}$ & $\begin{array}{l}0.08 \\
08\end{array}$ & $\begin{array}{l}0.591 \\
7 * * *\end{array}$ & $\begin{array}{l}0.472 \\
0 * * *\end{array}$ & $\begin{array}{l}0.497 \\
9 * * *\end{array}$ & $\begin{array}{l}- \\
0.364 \\
3^{*} \\
\end{array}$ & $\begin{array}{l}0.364 \\
8^{*}\end{array}$ \\
\hline & & $\mathrm{G}$ & & 1 & $\begin{array}{l}0.5 \\
430\end{array}$ & $\begin{array}{l}0.785 \\
7\end{array}$ & $\begin{array}{l}0.765 \\
4\end{array}$ & $\begin{array}{l}0.870 \\
1\end{array}$ & $\begin{array}{l}0.28 \\
90\end{array}$ & $\begin{array}{l}0.592 \\
9\end{array}$ & $\begin{array}{l}0.486 \\
7\end{array}$ & $\begin{array}{l}0.680 \\
0\end{array}$ & $\begin{array}{l}- \\
0.403 \\
3\end{array}$ & $\begin{array}{l}0.572 \\
5\end{array}$ \\
\hline \multirow[t]{2}{*}{3} & \multirow[t]{2}{*}{$\begin{array}{l}\text { Plant } \\
\text { height }\end{array}$} & $\mathrm{P}$ & & & 1 & $\begin{array}{l}0.297 \\
7 *\end{array}$ & $\begin{array}{l}0.237 \\
2\end{array}$ & $\begin{array}{l}0.485 \\
0 * * *\end{array}$ & $\begin{array}{l}0.31 \\
52^{*}\end{array}$ & $\begin{array}{l}0.192 \\
4\end{array}$ & $\begin{array}{l}0.226 \\
8\end{array}$ & $\begin{array}{l}0.310 \\
1^{*}\end{array}$ & $\begin{array}{l}- \\
0.280 \\
2\end{array}$ & $\begin{array}{l}0.444 \\
2 * *\end{array}$ \\
\hline & & $\mathrm{G}$ & & & 1 & $\begin{array}{l}0.531 \\
3\end{array}$ & $\begin{array}{l}0.518 \\
6\end{array}$ & $\begin{array}{l}0.505 \\
1\end{array}$ & $\begin{array}{l}0.70 \\
67\end{array}$ & $\begin{array}{l}0.532 \\
9\end{array}$ & $\begin{array}{l}0.625 \\
7\end{array}$ & $\begin{array}{l}0.630 \\
4\end{array}$ & $\begin{array}{l}- \\
0.503 \\
8\end{array}$ & $\begin{array}{l}0.542 \\
3\end{array}$ \\
\hline \multirow[t]{2}{*}{4} & \multirow{2}{*}{$\begin{array}{l}\text { Numb } \\
\text { er of } \\
\text { tillers } \\
\text { per } \\
\text { plant }\end{array}$} & $\mathrm{P}$ & & & & 1 & $\begin{array}{l}0.605 \\
2 * * *\end{array}$ & $\begin{array}{l}0.572 \\
3 * * *\end{array}$ & $\begin{array}{l}0.17 \\
39\end{array}$ & $\begin{array}{l}0.560 \\
8^{* * *}\end{array}$ & $\begin{array}{l}0.552 \\
3 * * *\end{array}$ & $\begin{array}{l}0.566 \\
7 * * *\end{array}$ & $\begin{array}{l}- \\
0.714 \\
0 * * *\end{array}$ & $\begin{array}{l}0.656 \\
3 * * *\end{array}$ \\
\hline & & $\mathrm{G}$ & & & & 1 & $\begin{array}{l}0.027 \\
8\end{array}$ & $\begin{array}{l}0.677 \\
3\end{array}$ & $\begin{array}{l}0.25 \\
93\end{array}$ & $\begin{array}{l}0.912 \\
9\end{array}$ & $\begin{array}{l}0.763 \\
3\end{array}$ & $\begin{array}{l}0.745 \\
3\end{array}$ & $\begin{array}{l}- \\
0.813 \\
9\end{array}$ & $\begin{array}{l}0.826 \\
4\end{array}$ \\
\hline \multirow[t]{2}{*}{5} & \multirow[t]{2}{*}{$\begin{array}{l}\text { Flag } \\
\text { leaf } \\
\text { length }\end{array}$} & $\mathrm{P}$ & & & & & 1 & $\begin{array}{l}0.615 \\
2 * * *\end{array}$ & $\begin{array}{l}0.20 \\
45\end{array}$ & $\begin{array}{l}0.672 \\
9 * * *\end{array}$ & $\begin{array}{l}0.504 \\
7 * * *\end{array}$ & $\begin{array}{l}0.478 \\
0 * * *\end{array}$ & $\begin{array}{l}- \\
0.589 \\
9 * * *\end{array}$ & $\begin{array}{l}0.527 \\
5 * * *\end{array}$ \\
\hline & & $\mathrm{G}$ & & & & & 1 & $\begin{array}{l}0.819 \\
9\end{array}$ & $\begin{array}{l}0.64 \\
02\end{array}$ & $\begin{array}{l}0.908 \\
7\end{array}$ & $\begin{array}{l}0.540 \\
0\end{array}$ & $\begin{array}{l}0.529 \\
5\end{array}$ & $\begin{array}{l}- \\
0.736 \\
8\end{array}$ & $\begin{array}{l}0.708 \\
7\end{array}$ \\
\hline \multirow[t]{2}{*}{6} & \multirow[t]{2}{*}{$\begin{array}{l}\text { Flag } \\
\text { leaf } \\
\text { width }\end{array}$} & $\mathrm{P}$ & & & & & & 1 & $\begin{array}{l}0.26 \\
78\end{array}$ & $\begin{array}{l}0.689 \\
4 * * *\end{array}$ & $\begin{array}{l}0.409 \\
8 * *\end{array}$ & $\begin{array}{l}0.544 \\
8 * * *\end{array}$ & $\begin{array}{l}- \\
0.502 \\
9 * * *\end{array}$ & $\begin{array}{l}0.562 \\
2 * * *\end{array}$ \\
\hline & & $\mathrm{G}$ & & & & & & 1 & $\begin{array}{l}0.64 \\
46\end{array}$ & $\begin{array}{l}0.758 \\
5\end{array}$ & $\begin{array}{l}0.628 \\
2\end{array}$ & $\begin{array}{l}0.635 \\
4\end{array}$ & $\begin{array}{l}- \\
0.637 \\
1 \\
\end{array}$ & $\begin{array}{l}0.738 \\
9\end{array}$ \\
\hline
\end{tabular}




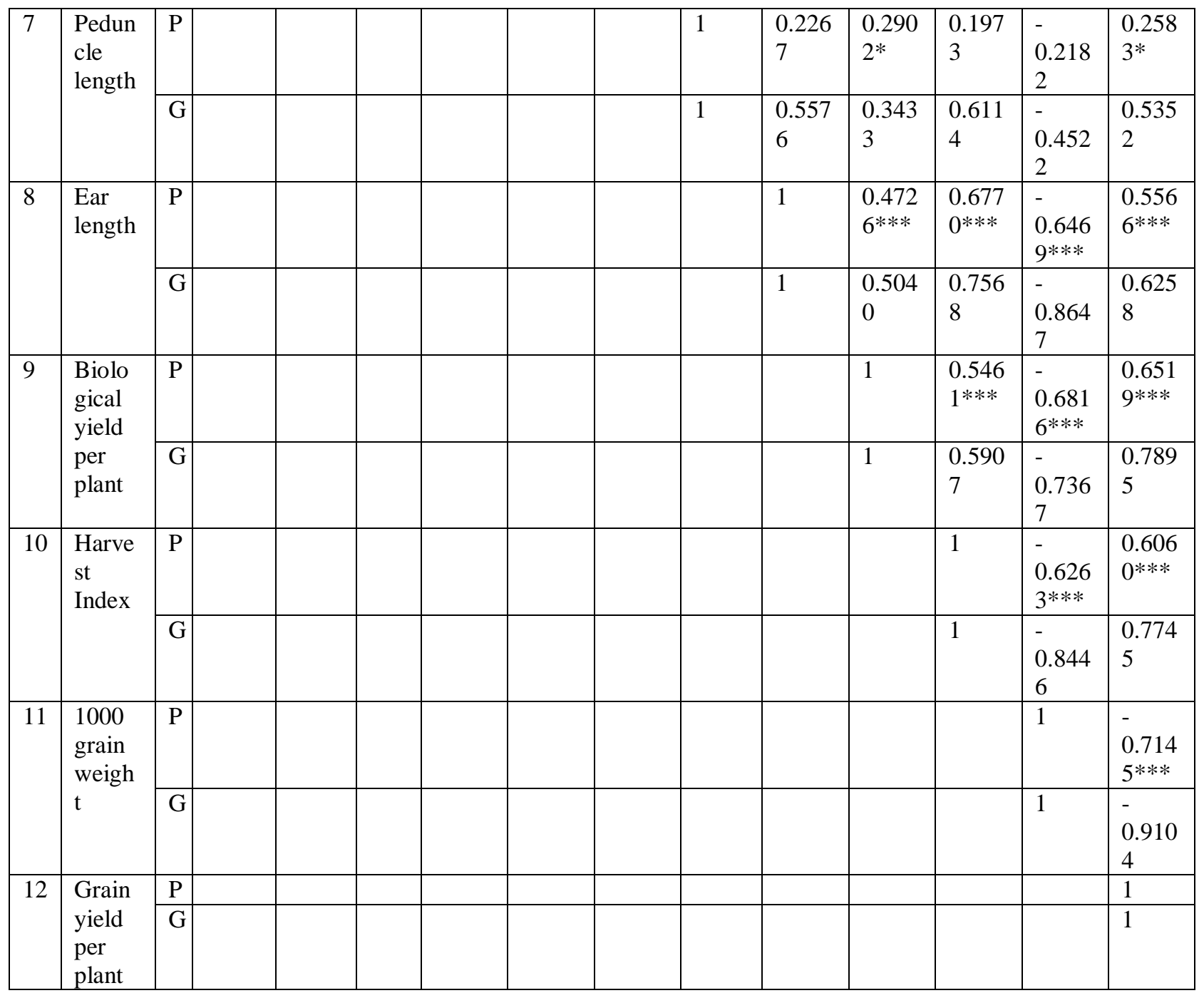

\section{References:-}

1. Gurunadharao Y and Apparao P. 1984. Genetic variability in yield and certain yield components of Italian millet.Madras Agricultural Journal. 71: 332-33.

2. Islam M, Mannujan H and Sarkar S.1990. An evaluation of Foxtail Millet germplasm in Bangladesh. Bangladesh Journal of Plant Breeding andGenetics. 2:59-61.

3. Nirmalakumari A, Ganapathy S and Murugan R. 2008. Studies on variability and descriptive statistics in Foxtail Millet germplasm .Crop Research. 35: 80-82.

4. Panse VG and Shukhatme PV. 1967. Statistical methods for agriculture workers . Indian Council of Agricultural Research, New Delhi.

5. Patil MS and Mohan Kumar HD. 1989. Studies on Genetic variability for yield and its components in Foxtail Millet. KarnatakaJournal Agricultural Sciences. 2(3): 165-169.

6. Prasanna PL, Murthy JSVS, Kumar PVR and Rao SV. 2013. Nature ofgene action for yield and yield components in exotic genotypes of Italian millet. Journal of Plant Breeding and Crop Science. 5(5): 80-84.

7. Velzaco JG and Rimieri P. 2012. Genotypic variability and response to selection in Foxtail Millet. Acta Agronomica. 61(3): 224-231. 